\title{
Growth and Investigation of Heterostructures Based on Multiferroic $\mathrm{BiFeO}_{3}$
}

B. Vengalis ${ }^{a, *}$, J. Devenson $^{a}$, A.K. Oginskis ${ }^{a}$, R. Butkuté ${ }^{a}$, A. Maneikis ${ }^{a}$, A. SteikŪniené $\dot{S}^{a}$, L. Dapkus ${ }^{a}$, J. Banys $^{b}$ AND M. KINKA ${ }^{b}$

${ }^{a}$ Semiconductor Physics Institute, A. Goštauto 11, LT-01108 Vilnius, Lithuania

${ }^{b}$ Vilnius University, Physics Department, Saulètekio al. 9, LT-10222 Vilnius, Lithuania

We report heteroepitaxial growth of multiferroic $\mathrm{BiFeO}_{3}$ thin films by RF magnetron sputtering on lattice-matched $\mathrm{SrTiO}_{3}$ substrates, as well as preparation and electrical properties of the heterostructures formed by growing $\mathrm{BiFeO}_{3}$ thin films on highly conductive $\mathrm{LaNiO}_{3}$ films and $n$-Si substrates. Nonlinear and rectifying current-voltage $(I-U)$ characteristics were revealed for the heterojunctions in a wide temperature range $(T=78-300 \mathrm{~K})$.

PACS numbers: 71.30.+h, 73.43.Qt, 75.47.Lx

\section{Introduction}

$\mathrm{BiFeO}_{3}$ (BFO) with a pseudo-cubic unit-cell parameter $(a \approx 3.96 \AA)$ of a rhombohedrally distorted perovskite structure belongs to a new emerging family of magnetic materials (multiferroics). A unique property of multiferroics is a coexistence of several ferroic orders (e.g. magnetic and electric) and some coupling between them (the magnetoelectric effect) [1, 2]. BFO is a ferroelectric below $1103 \mathrm{~K}$ and slightly canted antiferromagnet below $643 \mathrm{~K}$ [3]. Due to unusually high ordering temperatures, the $\mathrm{BFO}$ thin films are very promising for the fabrication of new types of devices for spintronics, ferroelectric memory applications, and sensors. For most of these applications, device structures composed of BFO thin films, conducting lattice-matched oxides and semiconductors such as Si and Ga might be elaborated. Further, electrical conductivity of BFO needs to be reduced for ferroelectric memory applications. Thus, there is great need to investigate the origin of electric conductivity and doping of the oxide [4].

*corresponding author; e-mail: veng@pfi.lt 
In this work, we are focusing on growth and properties of both epitaxial $\mathrm{BiFeO}_{3}$ thin films grown on lattice-matched $\mathrm{SrTiO}_{3}$ (STO) (100) substrates $(d=$ 50-200 nm) and related heterostructures formed by growing the BFO films on conducting $\mathrm{LaNiO}_{3}$ (LNO) thin films and highly doped $n$-Si(111) substrates.

\section{Results and discussion}

The BFO thin films with typical thickness $d$ ranging from about $150 \mathrm{~nm}$ to $200 \mathrm{~nm}$ were grown in situ at $T=750^{\circ} \mathrm{C}$ by $\mathrm{RF}$ magnetron sputtering (MS) on lattice-matched $\mathrm{SrTiO}_{3}(100)$, conducting $n$-Si(111) substrates $(\rho=0.01 \Omega \mathrm{cm})$ and $\mathrm{LaNiO}_{3}$ thin films. The disk-shaped ceramic target with excess Bi content $\left(\mathrm{Bi}_{1.1} \mathrm{FeO}_{3}\right)$ was synthesized to compensate possible loss of highly volatile Bi during $\mathrm{BFO}$ film growth. The native $\mathrm{SiO}_{2}$ oxide was removed from $\mathrm{Si}$ substrates by chemical etching just before BFO film deposition. Highly conductive LNO thin films were grown by DC magnetron sputtering at $T=750^{\circ} \mathrm{C}$ on $n$-Si(111) substrates using stoichiometric ceramic $\mathrm{LaNiO}_{3}$ target. After deposition, both the $\mathrm{BFO}$ and LNO thin films were annealed in pure oxygen at $T=600^{\circ} \mathrm{C}$ followed by slow cooling down to room temperature. Electrical properties of the grown BFO films were investigated by passing DC current perpendicular to a film plane. Metallic In pads attached mechanically to BFO, LNO film surface as well as to a bottom side of $n$-Si substrates were used as electrodes.

Investigation of X-ray diffraction (XRD) spectra revealed single-phase BFO films of epitaxial quality when grown on lattice-matched $\operatorname{SrTiO}_{3}(100)$ (see Fig. 1). At the same time, polycrystalline $\mathrm{BFO} / \mathrm{Si}(111)$ films with randomly oriented grains and preferentially (100)-axis oriented $\mathrm{BFO} / \mathrm{LNO} / \mathrm{Si}(111)$ films have been grown under the same deposition conditions. Surface of the grown films was studied by atomic force microscopy (AFM). The BFO films deposited onto the STO substrates showed relatively smooth surface with average grain size of about $200 \mathrm{~nm}$ and surface roughness of about $5 \mathrm{~nm}$ while slightly smaller grains and higher surface roughness values (of 12 and $9 \mathrm{~nm}$, respectively) have been indicated for the $\mathrm{BFO} / \mathrm{Si}$ and $\mathrm{BFO} / \mathrm{LNO} / \mathrm{Si}$ films.

Figure $2 \mathrm{a}$, b shows the current versus voltage $(I-U)$ characteristics measured for the $\mathrm{BFO} / \mathrm{LNO} / n$-Si (a) and $\mathrm{BFO} / n$-Si (b) heterostructures at $T=300 \mathrm{~K}$ and $78 \mathrm{~K}$. Positive $U$ values in the figure correspond to a positive voltage on the BFO overlayer. Nonlinear $I-U$ relationships have been found for both the heterostructures in a wide temperature range $(T=78-300 \mathrm{~K})$. Certain asymmetry of the $I-U$ curves in respect of current direction change can be noticed from Fig. 2a for the BFO/LNO/n-Si heterostructure, while clearly defined rectifying behavior (typical of semiconductor $p-n$ diode structures) have been indicated for the $\mathrm{BFO} / n$-Si heterostructure.

The observed $I-U$ relationships may be understood assuming $p$-type electrical conductivity of BFO films resulting formation of Schottky type barrier between BFO and highly hole-doped LNO while excellent rectifying properties of 


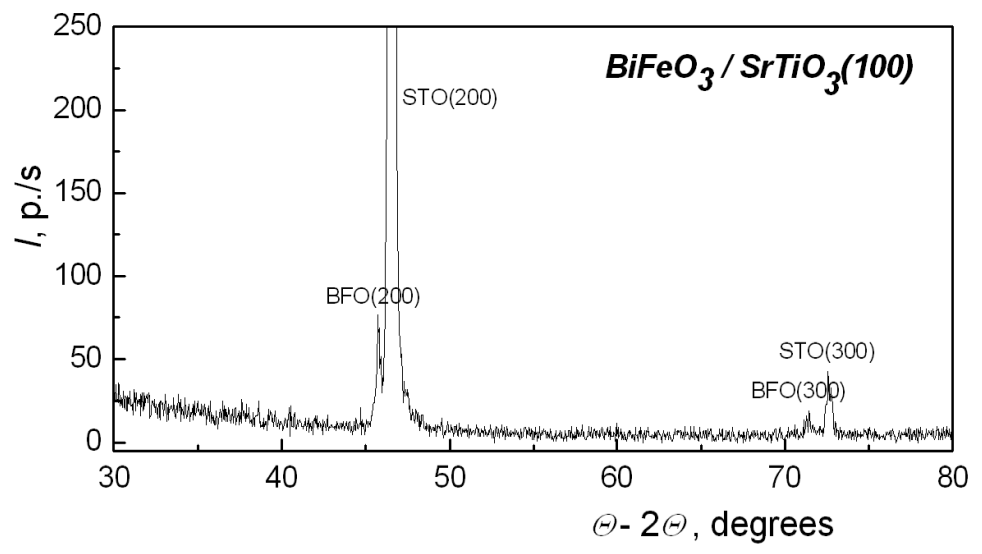

Fig. 1. $\Theta-2 \Theta$ XRD scan of the BFO film grown by $\mathrm{MS}$ on $\mathrm{SrTiO}_{3}(100)$ substrate.

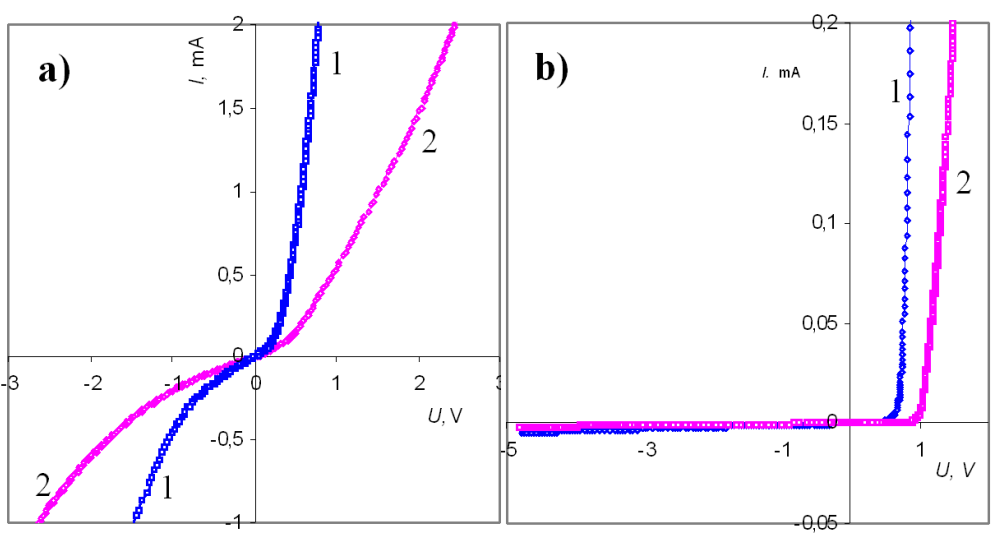

Fig. 2. Current versus voltage $(I-U)$ characteristics measured for the $\mathrm{BFO} / \mathrm{LNO} / n$-Si (a) and $\mathrm{BFO} / n$-Si (b) heterostructures at $T=300 \mathrm{~K}(1)$ and $78 \mathrm{~K}(2)$.

the $\mathrm{BFO} / n$-Si heterojunction may be expected due to the formation of a depletion layer with reduced carrier density in both $n$ - and $p$-type layers at the interface. Following Fig. 2a it is worth noting an increase in the diffusion voltage, $U_{\mathrm{D}}$ (corresponding to a step-like current growth in a case of a forward bias) for the $\mathrm{BFO} / n$-Si heterostructure from about 0.7 to $0.9 \mathrm{~V}$ with temperature decreasing down to $78 \mathrm{~K}$ in accordance to similar properties reported by us earlier for the heterostructures formed between $n$-Si and $p$-type manganites.

The $\log -\log$ plots of the $I-U$ curves shown in Fig. $2 \mathrm{a}$, b for the forward and reverse current directions are displayed in Fig. 3a, b. Following this figure we point out almost linear $I-U$ relationships for the heterostructures at low voltage values $(U \leq 0.1 \mathrm{~V})$ followed by an increased $I$ growth $\left(I \propto U^{\alpha}, \alpha=2 \div 7\right)$ in the intermediate region and certain saturation behavior at the highest voltage values. 

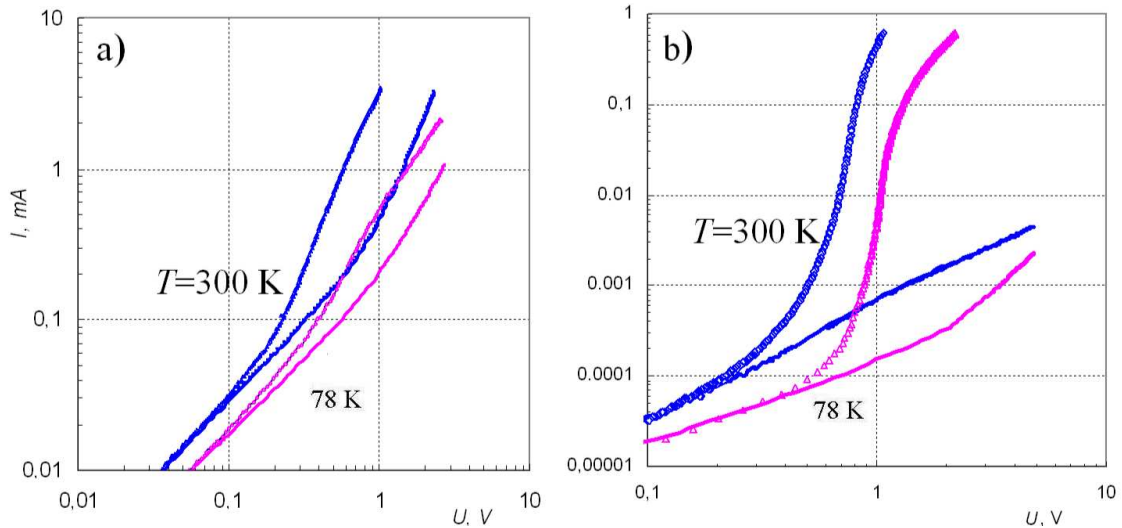

Fig. 3. Log- $\log$ plots of the $(I-U)$ characteristics measured for the $\mathrm{BFO} / \mathrm{LNO} / n-\mathrm{Si}$ (a) and $\mathrm{BFO} / n$-Si (b) heterostructures at $T=300 \mathrm{~K}$ and $78 \mathrm{~K}$ in a case of forward and reverse bias.

We point out finely presence of $p$-type electrical conductivity of BFO thin films, nonlinear $I-U$ relations of the $\mathrm{BFO} / \mathrm{LNO}$ heterostructure due to the formation of Schottky type barrier at the interface and clearly defined rectifying properties of the $\mathrm{BFO} / n$-Si heterojunctions.

\section{Acknowledgments}

The work was partly supported by the Lithuanian State Science and Studies Foundation (grants No. T-102/07 and C-18/2007).

\section{References}

[1] N.A. Hill, J. Phys. Chem. B 104, 6694 (2000).

[2] G.A. Smolenskii, I.E. Chupis, Sov. Phys. Usp. 25, 475 (1982).

[3] J. Wang, J.B. Neaton, H. Zheng, V. Nagarajan, S.B. Ogale, B. Liu, D. Vehland, V. Vaithyanathan, D.G. Schlom, U.V. Waghmare, N.A. Spaldin, K.M. Rabe, M. Wuttig, R. Ramesh, Science 299, 1719 (2003).

[4] X. Qi, J. Dho, R. Tomov, M.G. Blamire, J.L. Mac- Manus-Driscol, Appl. Phys. Lett. 86, 062903 (2005). 